\title{
Research on Reliability of Aeroengine based on Grey Correlation Algorithm
}

\author{
Jian Zhang ${ }^{a}$, Yanjun $\mathrm{Li}^{\mathrm{b}}$, Lina Zhang ${ }^{\mathrm{c}}$, Zhengqiang Cheng ${ }^{\mathrm{d}}$ \\ College of Civil Aviation, Nanjing University of Aeronautics and Astronautics, Nanjing 211106,China \\ aemail: 1540079310@qq.com, bemail: Iyj@nuaa.edu.cn, \\ cemail: 747931881@qq.com, ${ }^{\mathrm{d}}$ email: 269102349@qq.com
}

\section{Keywords: Aeroengine; Gray Correlation Algorithm; Attribute Reduction; Reliability}

\begin{abstract}
With the rapid development of the aviation industry, the safety of aircraft is obtaining more and more attention. Engine is the key component of the aircraft, its reliability is increasingly important. This paper proposes a new method of reliability analysis for aircraft engine using grey correlation algorithm based on its characteristic of studying system that some information is known, some information is unknown, small sample, poor information or uncertain. Firstly, simplify the performance parameters of aeroengine using gray relational clustering algorithm to extract key parameters. Then, calculate the reliability of aeroengine using grey relational degree algorithm. Finally, verify the algorithm proposed in this paper using the data of Honeywell. The results show that the algorithm proposed in this paper can effectively estimate the reliability of aeroengine.
\end{abstract}

\section{Introduction}

Aeroengine fault occupies a large proportion in flight failure and is the main factor affecting flight safety. Aeroengine is expensive and complex, its working conditions is harsh, so the reliability is hard to guarantee. Therefore, it has great significance to implement timely condition monitoring and fault diagnosis.[1] Traditional fault diagnosis methods compare the measured data with calculated data from standard model and distinguish the reliability of engine according to the size of difference.

After decades of development, there are a large number of fault diagnosis methods, mainly divided into three categories:1) Methods based on analytical model, such as state estimation method, spatial equivalent method, parameter estimation methods, etc. 2) Methods based on signal processing, such as spectral analysis, wavelet analysis, correlation analysis, etc. 3) Methods based on knowledge, such as intelligent diagnosis, neural networks, fuzzy reasoning, etc.[2]

At present, the intelligent method mainly used are neural networks [3][4] and expert systems[5], but there all have shortness. Such as, neural networks have the problems of the selection of network structure and are easy to fall into local optima; expert system has the problems of incomplete knowledge and poor adaptability. The gray theory studies system that some information is known, some information is unknown, small sample, poor information or uncertain and extract valuable information by generating or developing known information to achieve the correct description of the operating rules and effective control [6].

Therefore, this paper proposes a method that uses gray correlation algorithm to extract key parameters and calculate reliability of aeroengine as a reference and guidance for engine safety.

\section{Attribute Reduction of Aeroengine based on Grey Relational Clustering}

The attribute reduction algorithm based on grey relational clustering is proposed in this paper. According to the dependence of the engine parts on different performance parameters in different failure modes, the performance parameters of a failure mode can be selected, which can be used as the input of prediction and diagnosis system. This method uses the gray correlation analysis method 
to calculate the correlation degree matrix of various failure modes, and then introduces the calculation method of grey correlation degree in dynamic clustering.

$n$ represents the number of performance parameters. Each parameter in different fault mode is measured by the normalized data $x_{i}(k)(i=1,2, \cdots, n ; k=1,2, \cdots, m), m$ is the fault type, the parameter of fault state sequence is $X_{i}=\left(x_{i}(1), x_{i}(2), \cdots, x_{i}(m)\right)$.

The calculation method of the correlation degree between the parameters $i$ and $j$ in the fault mode $k$ is as follows:

(1) Calculate the sequence difference $\Delta_{i}(k)=\left|x_{j}(k)-x_{i}(k)\right|(i, j=1,2, \cdots, n ; k=1,2, \cdots, m)$ get $\Delta_{i}=\left(\Delta_{i}(1), \Delta_{i}(2), \cdots, \Delta_{i}(m)\right)$

(2) Find the maximum difference $M$ and the minimum difference $m$ $M=\max _{i} \max _{k} \Delta_{i}(k), m=\min _{i} \min _{k} \Delta_{i}(k)$

(3) Calculate the correlation coefficient:

$$
\gamma_{0 i}(k)=\frac{m+\xi M}{\Delta_{i}(k)+\xi M}(\text { 耳又 } \xi=0.5), i=1,2, \cdots, n ; k=1,2, \cdots, m
$$

(4) Calculate associate degree:

$$
\gamma_{0 i}=\frac{1}{m} \sum_{k=1}^{m} \gamma_{i j}(k) ;(i=1,2, \cdots, n)
$$

The correlation degree $r_{i j}$ reflects the degree of correlation between parameters $X_{j}$ and parameters $X_{i}$. The correlation degree of a parameter with other parameters is $r_{i}=\left(r_{i 1}, r_{i 2}, \cdots, r_{i n}\right)$.

Clustering is the process of dividing $n$ performance parameters into $k$ classes. Each group contains at least one object, and each object must be classified into a group. In a failure mode, the grey incidence matrix composed of $\mathrm{n}$ performance parameters is $R$, and the generalized grey Euclidean distance between objects $X_{i}$ and cluster center $m_{k}$ is

$$
d_{i}=d\left(X_{i}, m_{k}\right)=\left|\frac{1}{\sum_{j=1}^{n} r_{i j}}-m_{k}\right|
$$

If the number of clustering is $k$, the specific steps of the grey relational degree clustering algorithm are as follows [7]:

1) Select a parameter $m_{1}$ as the cluster center in a series of failure mode, and then calculate the distance of remaining $N-1$ parameters $r_{1 j}^{1}$ with $m_{1}$. Here define the distance between parameters ' $\mathrm{i}$ ' and ' $\mathrm{j}$ ' as the reciprocal of correlation and choose the parameter composing of the maximum distance as the cluster center of second type that is $m_{2}$;

2) Calculate the distance of the remaining $N-2$ parameters with $m_{1}$ and $m_{2}$, choose the parameter composing of the maximum distance as cluster center of third type that is $m_{3}$;

3) Choose the point that farthest with all cluster centers as the next cluster center. Continue to calculate until the number of cluster center reaches $K$.

4) Classify all other parameters into their nearest cluster center category and realize the classified parameters;

5) In each category, calculate $\sum_{j=1}^{n} \mathrm{r}_{i j}$ of each parameter, choose the maximum value $R_{i}$ of each category as characteristic parameters of the model;

\section{Reliability Analysis of Aero-engine based on Grey Correlation Degree}

Reliability refers to the ability of completing the required functions for product in the specified conditions, within the specified time and is a measure for the ability of working normally. Reliability analysis and evaluation is a very important part in reliability analysis. It refers to the process of estimating the reliability index of products based on the reliability distribution model, 
structure and related reliability information, using statistical methods [8][9].

For nearly a century, scholars have made great achievements in the field of reliability research. The reliability theory is getting more and more perfect, and the engineering application effect has been improved. However, the traditional reliability analysis based on large sample probability statistics, can only give the results of the analysis of statistical significance. Using traditional reliability analysis method to evaluate the operational reliability of aeroengine has the following two difficulties. Firstly, it is difficult to effectively deal with the small sample failure data based on the large sample condition. Aeroengine has characteristics of high reliability and long life. The reliability evaluation is a typical small sample problem. Secondly, the degradation form of aeroengine is different under different operating conditions, and the accuracy of reliability evaluation to the single equipment using the traditional reliability method based on the common failure law of large samples is low[10].

The specific steps of the reliability evaluation method of aeroengine based on grey correlation degree are as follows:

(1) Frame vector $X=(x(1), x(2), \cdots, x(n))$ with baseline value of the aircraft engine, $x(n)$ is the performance parameter representing state.

(2) Frame the corresponding pattern vector $Y=(y(1), y(2), \cdots, y(n))$ according to the engine condition,.

(3) Choose the base value $X$ of the step (1) as a characteristic vector and the vector $Y$ of the step (2) as the vector. Calculate the initial value of each vector

$$
\left\{\begin{array}{l}
X^{\prime}=X / x(1)=x^{\prime}(1), x^{\prime}(1), \cdots, x^{\prime}(n) \\
Y^{\prime}=Y / y(1)=y^{\prime}(1), y^{\prime}(1), \cdots, y^{\prime}(n)
\end{array}\right.
$$

(4) Calculate the sequence difference: $\Delta(k)=\left|y^{\prime}(k)-x^{\prime}(k)\right|(k=1,2, \cdots, n)$

(5) Find the maximum difference $M$ and the minimum difference $m$ $M=\max _{i} \max _{k} \Delta_{i}(k), m=\min _{i} \min _{k} \Delta_{i}(k)$

(6) Calculate correlation coefficient:

$$
\gamma_{0 i}(k)=\frac{m+\xi M}{\Delta_{i}(k)+\xi M}(\text { 取 }=0.5), i=1,2, \cdots, n ; k=1,2, \cdots, m
$$

(7) Calculate association: $\gamma_{0 i}=\frac{1}{m} \sum_{k=1}^{m} \gamma_{i j}(k) ;(i=1,2, \cdots, n)$

(8) The reliability of the state is related to the correlation in the step (7),because of $\gamma_{0}(k)=\frac{m+\xi M}{\Delta(k)+\xi M} \in\left[\frac{1}{3}, 1\right]$,the degree of correlation can be equivalent to reliability as follows:

$$
P=\frac{\gamma_{0}(k)}{1-\frac{1}{3}}=1.5 \gamma_{0}(k)
$$

\section{Instance verification}

In this paper, verify the accuracy of the algorithm with a certain type data of aeroengine provided by Honeywell. Construct parametric model with the parameters representing aeroengine that is Exhaust Gas Temperature (EGT), Fan Shaft Speed $\left(\mathrm{N}_{1}\right)$, Core Shaft speed $\left(\mathrm{N}_{2}\right)$,Fuel Flow (FF), Oil Temperature (OIT), Oil Pressure (OIP) as the system input parameters. Firstly, compare monitoring value with their baseline value and get the difference. The calculation formula is as follows: $\Delta x=\left(\left(x_{l}-x_{b}\right) / x_{b}\right) \times 100 \%$

In the formula, $x_{l}$ is the actual value of the parameters, $x_{b}$ is the parameters of the baseline value.

Choose 5 group of performance parameters of high pressure turbine typical faults (HPT), it is shown in table 1 after calculating with formula (9) 
Table 1 Train samples

\begin{tabular}{|c|c|c|c|c|c|c|}
\hline Mode & $\Delta \mathrm{EGT} / \%$ & $\Delta \mathrm{N}_{2} / \%$ & $\Delta \mathrm{OIP} / \%$ & $\Delta \mathrm{FF} / \%$ & $\Delta \mathrm{N}_{1} / \%$ & $\Delta \mathrm{OIT} / \%$ \\
\hline Working blade damage( I ) & -1.00 & -0.05 & -0.35 & -0.25 & -0.25 & -0.50 \\
\hline Outside sealing gap large( II ) & -2.00 & -0.05 & -0.25 & -0.40 & -0.20 & -0.35 \\
\hline Working blade wear(III) & -7.00 & 0.10 & -0.15 & -0.85 & -0.10 & -0.30 \\
\hline Guiding blade burned(IV) & -0.05 & -0.25 & -0.05 & -0.10 & 0.00 & -0.20 \\
\hline Guiding blade damage( V) & -8.50 & 0.20 & -0.10 & -1.05 & -0.10 & -0.15 \\
\hline
\end{tabular}

6 performance parameters are clustered into 3 classes, each parameter is used as the initial cluster center, and the clustering results obtained by the above method are shown in table 2

Table 2 Clustering results

\begin{tabular}{|l|l|l|}
\hline Starting center & Clustering results & Characteristic parameters \\
\hline EGT & EGT/ OIT/ OIP/ $N_{1} 、 \mathrm{~N}_{2} 、$ FF & $N_{2} / \mathrm{EGT} / \mathrm{FF}$ \\
\hline $\mathrm{N}_{2}$ & $\mathrm{~N}_{2} / \mathrm{OIT} / \mathrm{OIP} / \mathrm{N}_{1} 、 \mathrm{EGT} 、 \mathrm{FF}$ & $\mathrm{N}_{2} / \mathrm{EGT} / \mathrm{FF}$ \\
\hline $\mathrm{OIP}$ & OIP/EGT、 $\mathrm{N}_{1} / \mathrm{N}_{2} 、 \mathrm{OIT} / \mathrm{FF}$ & $\mathrm{N}_{2} / \mathrm{EGT} / \mathrm{FF}$ \\
\hline $\mathrm{FF}$ & $\mathrm{FF} / \mathrm{OIT} 、 \mathrm{~N}_{1} / \mathrm{N}_{2} 、 \mathrm{OIP} / \mathrm{EGT}$ & $\mathrm{N}_{2} / \mathrm{EGT} / \mathrm{FF}$ \\
\hline $\mathrm{N}_{1}$ & $\mathrm{~N}_{1} / \mathrm{N}_{2} 、 \mathrm{OIP} / \mathrm{EGT}$ OIT/FF & $\mathrm{N}_{2} / \mathrm{EGT} / \mathrm{FF}$ \\
\hline OIT & $\mathrm{OIT} / \mathrm{FF} 、 \mathrm{~N}_{1} / \mathrm{N}_{2} 、 \mathrm{EGT} / \mathrm{OIP}$ & $\mathrm{N}_{2} / \mathrm{EGT} / \mathrm{FF}$ \\
\hline
\end{tabular}

It can be found that the characteristic parameter is $N_{2} / E G T / F F$, and the selection of the initial cluster does not affect the selection of the characteristic parameters.

According to the attribute reduction, choose 1 group of performance parameters of the engine in the normal state, the slight fault condition and the critical failure state of the engine. The parameter values are shown in table 3:

Table 3 Verification sample

\begin{tabular}{llccc}
\hline No。 & Real state & $\mathrm{N}_{2}$ & EGT & FF \\
\hline 1 & baseline & 94.3125 & 593.5 & 2776 \\
2 & normal & 93.9063 & 597 & 2782 \\
3 & slight failure & 86.7813 & 612 & 2584 \\
4 & critical failure & 85.3125 & 623 & 2904 \\
\hline
\end{tabular}

The performance degradation curve of this type of engine is shown in Figure 1:

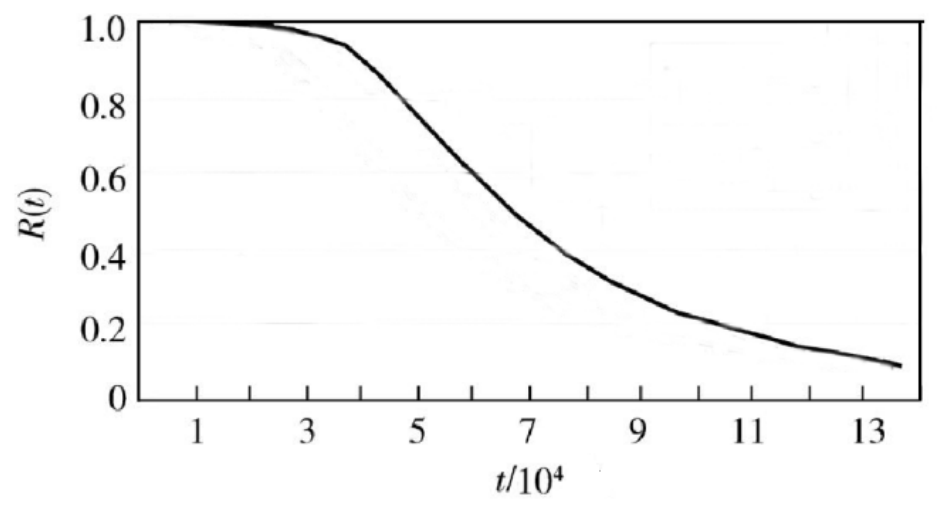

Fig.1. Performance degradation curve

According to the algorithm proposed in this paper, calculate the reliability of the four states in Table 3.The corresponding flight cycle times and the actual reliability are shown in Table 4:

\section{Table 4 Verification results}

\begin{tabular}{llll}
\hline Cycle-index & Actual & reliability/\% & Algorithm reliability /\% \\
\hline 100 & 100 & 100 \\
40000 & 93 & 92.71 \\
85000 & 32 & 32.8 \\
100000 & 23 & 25.4 \\
\hline
\end{tabular}




\section{Conclusion}

The results show that the algorithm based on gray correlation algorithm can effectively reduce the engine performance parameters, extract the key parameters of the characterization performance, and evaluate the reliability of the engine, which can provide references for the monitoring and state reliability of aeroengine.

\section{Acknowledgement}

In this paper, the research was sponsored by Aeronautical Science Foundation of China (Project No. 201510301007131) and Nanjing University of Aeronautics and Astronautics School Open Fund (Project No. kfjj20150701).

\section{References}

[1] Yinghong Li, Xunkai Wei, Jianxun Liu. Application of support vector machine to engineering [M]. Beijing: Enginery Press, 2004.

[2] Linjun Li, Zhousuo Zhang, Zhengjia He. Application research on support vector machines in mechanical fault diagnosis [J]. Computer Engineering and Applications, 2002, 19:19-21.

[3] Chen T, Sun J G, Yang W H, et al. Self-organizing neural network based fault diagnosis for aero engine gas path [J]. Aeronautical journal. 2003(01):46-48.

[4] Tian Chen, Jianguo Sun, Ying Hao. Neural network and dempster-shafter theory basde fault diagnosis for aero engine gas path [J]. Aeronautical journal. 2006(06):1014-1017.

[5] Guo Chen, Hongfu Zuo. Expert systems of engine wear fault diagnosis based on knowledge rule[J].Journal of Aerospace Power,2004,19(1):23-29(in Chinese).

[6] Julong Deng. Grey system [M].Beijing: National Defence Industry Press, 1985.

[7] Feng Lu, Jinquan Huang. Feature extraction algorithm of clustering based on grey relational theory [J].Systems Engeering Theory and Practice, 2012，32(4): 872-876.

[8] Qizhi Mei. System reliability engineering foundation [M] .Beijing: Science Press, 1987.

[9] Shuhong Ren. Research on Methods of Performance Reliability Assessments and Life on Wing Prediction for Civil Aero engine [D]. Nanjing : Nanjing University of Aeronautics and Astronautics, 2010.

[10] Chuang Sun, Zhengjia He, Zhousuo Zhang, et al. Operating Reliability Assessment for Aero-engine Based on Condition Monitoring Information [J] . Journal of Mechanical Engineering, 2013, 49(6): 30-37. 